\begin{tabular}{|c|l|}
\hline Title & Characterization of the interaction of influenza virus NS1 with A kt \\
\hline Author(s) & Matsuda, Mami; Suizu, Futoshi; Hirata, Noriy uki; Miy azaki, Tadaaki; Obuse, Chikashi; Noguchi, Masay uki \\
\hline Citation & $\begin{array}{l}\text { Biochemical and Biophysical Research Communications, 395(3), 312-317 } \\
\text { https://doi.org/10.1016/.bbrc.2010.03.166 }\end{array}$ \\
\hline Issue Date & 2010-05-07 \\
\hline Doc URL & http://hdl.handle.net/2115/43121 \\
\hline Type & article (author version) \\
\hline File Information & BBRC395-3_312-317.pdf \\
\hline
\end{tabular}

Instructions for use 


\section{Characterization of the interaction of influenza virus NS1 with Akt}

Mami Matsuda\#, Futoshi Suizu", Noriyuki Hirata ${ }^{\#}$, Tadaaki Miyazaki ${ }^{\mathrm{p}}$,Chikashi Obuse ${ }^{\Psi}$, and Masayuki Noguchi ${ }^{\# \S}$

\# Division of Cancer Biology, Institute for Genetic Medicine, Hokkaido University, Sapporo, Japan

P Department of Bioresources, Hokkaido University Research Center for Zoonosis Control, Sapporo, Japan

$\Psi$ Division of Molecular Life Science, Graduate School of Life Science, Hokkaido University, Sapporo, Japan

${ }^{\S}$ Correspondence should be addressed. Masayuki Noguchi, MD, PhD

N15, W7, Kita-ku, Sapporo 060-0815, Japan

TEL: +81-11-706-5069; FAX: +81-11-706-7826;

E-Mail: m_noguch@igm.hokudai.ac.jp 


\begin{abstract}
Avian influenza viruses belong to the genus influenza A virus of the family

Orthomyxoviridae. The influenza virus consists of eight segmented minus stranded RNA that encode 11 known proteins. Among the 11 viral proteins, NS1 (non-structural protein 1 , encoded on segment 8 ) has been implicated in the regulation of several important intra-cellular functions.
\end{abstract}

In this report, we investigated the functional interaction of NS1 with serine threonine kinase Akt, a core intra-cellular survival regulator. In co-immunoprecipitation assays and GST pull-down assays, NS1 directly interacted with Akt. The interaction was mediated primarily through the Akt-PH (Pleckstrin Homology) domain and the RNAbinding domain of NS1. NS1 preferentially interacted with phosphorylated Akt, but not with non-phosphorylated Akt. Functionally, the NS1-Akt interaction enhanced Akt kinase activity both in the intra-cellular context and in in vitro Akt kinase assays. Confocal microscopic analysis revealed that phosphorylated Akt interacted with NS1 during the interphase of the cell cycle predominantly within the nucleus. Finally, mass spectrometric analysis demonstrated the position at Thr215 of NS1 protein is primary phosphorylation target site through Akt activation. The results together supported the functional importance of influenza virus NS1 with Akt, a core intra-cellular survival regulator. 


\section{Keywords}

Influenza virus

Non-structural Protein 1 (NS1)

Akt

\section{Abbreviations}

NS1: Non Structural protein 1

PH domain : Pleckstrin Homology domain

PI3K: Phosphoinositide 3- kinases

RBD: RNA Binding Domain

EFF : Effector Domain 


\section{Introduction}

In recent years, pandemic influenza of $\mathrm{H} 1 \mathrm{~N} 1$ avian influenza viruses have emerged and now appear to spread in many regions throughout the world. Avian influenza viruses belong to the genus influenza A virus of the family Orthomyxoviridae. Based on the antigenic specificity, influenza A viruses are divided into $16 \mathrm{HA}$ and nine neuramidase (NA) subtypes. The influenza virus consists of eight segmented minus stranded RNA that encode 11 known proteins. Among the 11 viral proteins, NS1 (non-structural protein 1 , encoded on segment 8) has been implicated in the regulation of several biological functions, including regulation of apoptosis suppression of host immune responses, inhibition of nuclear export of mRNA and splicing of mRNA by binding U6 small nuclear RNA(snRNA) [1]. NS1 has also been shown to bind to double-strand RNA and, as a consequence, inhibits PKR kinase activity[2]. Functional connection of influenza A virus NS1 with cellular apoptosis through NF-kB-dependent SOCS-3 in which JAK-STAT, MAPK, and PI3K cooperatively immunomodulate IFN responses has also been reported [3].

Upon infection by the influenza A virus, varieties of intracellular signaling pathways are known to be activated. The PI3K-Akt network has recently been demonstrated to underlie clinical manifestations of various stages of viral infection, such as acute 
infection, latent infection, or chronic infection of the Epstein-Barr virus, the hepatitis $\mathrm{C}$ virus, the hepatitis B virus, the human immunodeficiency virus, and the influenza virus $[4 ; 5]$.

Serine threonine kinase Akt is activated in response to PI3K by a wide variety of growth factors, antigens, and inflammatory stimuli. Activation of PI3K produces PtdIns $(3,4,5) \mathrm{P}_{3}\left(\mathrm{PIP}_{3}\right)$, which binds to the lipid-binding module of the $\mathrm{PH}$ domain of Akt, and induces activation and its downstream signals to regulate a wide variety of cellular responses, including anti-apoptosis, proliferation, cell cycling, protein synthesis, glucose metabolism, and telomere activity[6].

The binding of $\mathrm{PIP}_{3}$ to the Akt-PH domain and subsequent translocation of Akt to the plasma membrane allow PDK1 to access and trigger phosphorylation at a threonine residue (Thr308/305) within the catalytic domain of Akt. TORC2 (mTOR-Rictor complex) has recently been shown to be major activation mechanism for the phosphorylation of Ser473 of Akt in the carboxy-terminal hydrophobic motif. Activation of Akt promotes anti-apoptotic activity by preventing the release of cytochrome c from mitochondria and inactivating FOXO families to induce expression of proapoptotic factors such as FAS ligand.

NS1 protein of influenza A virus interacts with varieties of cellular multifunctional 
protein during infection $[1 ; 7]$. In this report, we investigated the physical interaction of influenza NS1 protein in the host signaling cascade, particularly the functional connection with Akt, a core intra-cellular survival regulator. The therapeutic implications of this observation are also discussed. 


\section{Material and methods}

\subsection{Co-immunoprecipitation experiments}

Co-immunoprecipitation experiments were essentially performed as described previously [8]. In brief, 293T cells (ATCC) were co-transfected with a total of $7.5 \mu \mathrm{g}$ of indicated plasmids per $10 \mathrm{~cm}$ dish. Seventy-two hours after transfection, cells were harvested, and lysed with Brij 97 lysis buffer with proteinase inhibitors (Leupeptin, and AEBSF). Lysates were precleaned with ProA/G beads (50\% v/v, Amersham, [8]) for $1 \mathrm{~h}$, immunoprecipitated with anti-HA or anti-Flag antibody (or other indicated antibodies) with mouse IgG as a control, run on SDS-PAGE, and immunoblotted with indicated antibodies.

\subsection{Interaction of Akt with NS1 in PR8 (H1N1) infected A549 cells}

A549 cells were infected with influenza A/PR/8/34(H1N1) (PR8) at a M.O.I (multiplicity of infection) of 1 . Cell lysates were prepared at 17 hours. Post-infection (p.i), and immunoprecipitated with anti-Akt antibody (Cell Signaling) or mouse IgG (control, DAKO), and subjected to immunoblot using anti-NS1 antibody (SantaCruz) to determine the interaction. 


\subsection{GST pull-down assays of active/unactive form of Akt}

RNA binding domain (RBD) and Effector domain (EFF) of NS1 were PCR amplified and subcloned into pGEX4T-2 (Pharmacia). Then recombinant GST-NS1 (EFF or RBD) protein was generated according to the manufacture's protocol. Reaction mixtures [60ng recombinant each Akt (active/unactive Akt, \#14-276 active Akt1/PKB $\alpha$,

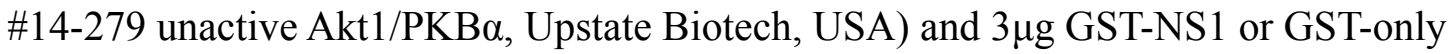
as a control] in Brij97 cell lysis buffer were incubated for $1 \mathrm{hr}$ at $4^{\circ} \mathrm{C}$ with gentle agitation. The resulted samples were washed three times and resolved onto SDS-PAGE, immunoblotted using anti pan-Akt antibody [Akt1/2(N-19), \#sc1619 SantaCruz] and detected by ECL.

\section{4. in vitro Akt kinase assays of NS1}

NS1 protein plasmid constructs were generated by PCR amplification and subcloned into the pGEX4T-2 vector (Pharmacia) or other indicated mammalian expression plasmids. In vitro kinase assays (IVK) were performed essentially using the Akt kinase assay kit (Cell Signaling) with minor modifications. Recombinant GST protein was added to adjust the total protein concentration constant in the kinase assays as shown in 
the CBB staining gel. HA-Akt1 from 293T cells transfected with pCMV-HA-Akt1 was immobilized with anti-HA antibody (12CA5) conjugated with ProA/ProG beads. IVK reaction was performed using GSK $3 \beta$ protein $(2.5 \mu \mathrm{g})$ as substrates by incubating in the presence of $2 \mu \mathrm{l}$ (approximately $5 \mathrm{ng}$ ) of immobilized Akt1 for 7 min at $30{ }^{\circ} \mathrm{C}$ with gentle agitation. The reactions were terminated by adding $2 \times$ SDS sample buffer. The samples were separated on SDS-PAGE and immunoblotted with phospho-Thr308 Akt, phosphoSer473 Akt or phospho-GSK3 $\beta$ antibodies (Cell Signaling) and detected by ECL.

\subsection{Co-localization experiment using a confocal microscopy}

HeLa cells (ATCC) were cultured in DMEM with 10\% FCS with penicillin and streptmycin, transfected with Ds-Red-tagged NS1 (Invitrogen) and/or indicated plasmid, fixed with 3.7\% formaldehyde, stained with indicated antibodies (anti-phospho-Ser473 Akt), or DAPI (4',6-diamidino-2-phenylindole, blue, Sigma), and examined using a confocal microscopy (Olympus).

\subsection{Identification of Akt-induced NS1 phosphorylation site by mass spectrometry}

Flag-tagged NS1 was transfected into 293T cells along with Myr-Akt, a constitutive active form of Akt, by calcium phosphate method described elsewhere. Seventy-two 
hours after the transfection, the cells were harvested and lysed with Brij97 lysis buffer, precleaned with ProA/ProG beads [8], and immunoprecipitated with Flag M2 beads (Sigma). Immunoprecipitated Flag-NS1 products were subjected to resolve onto SDS-PAGE. Gel slices corresponding to NS1 protein were excised and subjected to in-gel trypsinization to examine the phosphorylation site using MS/MS analysis by LXQ (Thermo Fisher) equipped with nano-flow LC Magic (Michrom Bioresources, Inc.). The MS/MS spectra of the tryptic amino acids were assigned by Mascot search (Matrix Science). The spectra for potential phosphorylated peptide were manually examined.

Antibodies used in this study are listed in the supplementary material. 


\section{Results}

\subsection{Influenza virus NS1 interacted with Akt in co-immunoprecipitation assays.}

The PI3K-Akt signaling network plays a central role in the regulation of intra-cellular anti-apoptotic action. In recent years, the PI3K-Akt signaling network has been shown to underlie the clinical manifestation of several stages of viral infection[4; $5]$.

Since NS1 has been shown to be involved in the regulation of anti-apoptosis and Akt is known to be one of the major intra-cellular survival regulators, we first asked whether NS1 can physically interact with Akt. We performed co-immunoprecipitation assays using $293 \mathrm{~T}$ cells transfected with HA-tagged human Akt2 and Flag-tagged NS1. HA-tagged Akt co-immunoprecipitated with Flag-tagged NS1 in this experiment (Fig. 1A).

We next examined whether NS1 protein interacts with endogenous Akt in influenza A/PR/8/34(H1N1)(PR8) infected cells. For this purpose, A549 cells were infected with PR8 and demonstrated that endogenous Akt indeed bound to NS1 in PR8-infected cells

(Fig. 1B). 
To define the interaction domains of Akt with NS1, we next performed co-immunoprecipitation assays using $293 \mathrm{~T}$ cells transfected with HA-tagged human full length Akt2, PH domain of Akt (PH), or C-terminal domain of Akt (C-term [8]) with Flag-tagged wild type NS1 protein. The Akt-PH-domain primarily mediated the interaction with NS1 shown by the co-immunoprecipitation assays (Fig. 1C). Phosphorylation is known to play an important role in the interaction of the intra-cellular molecules in various cellular environments [8]. Therefore, we next compared whether and how levels of Akt phosphorylation can affect its physical interaction with NS1 protein. Co-immunoprecipitation assays were performed using 293 cells transfected with Flag-tagged NS1 with either HA-tagged wild-type Akt or T308A/S473A(Double Mutant, DM)-Akt, in which both Thr308 and Ser473 of the critical phosphorylation residues of Akt were substituted into alanine (Fig. 1D). Wild-type Akt interacted more efficiently than DM-Akt with NS1. Consistent with this observation, K177M-Akt, in which ATP-binding sequences were destroyed by introducing the methionin at position 177 , or R25C-Akt, which lacks the ability to bind $\mathrm{PIP}_{3}$ failed to interact with wild-type NS1 (data not shown). Together, the results demonstrated that NS1 preferentially binds to phosphorylated Akt.

Next, we compared the interaction of the activated or inactivated form of Akt with 
NS1 by GST pull-down assays using activated and inactivated forms of recombinant Akt (active/unactive Akt, Upstate Biotech). NS1 interacted more efficiently with the active form of Akt but failed to interact with the inactive form of Akt in this assay (Fig. 1E).

The interaction between Akt and NS1 is primarily mediated through the RNA-binding domain (RBD) of NS1 in this assay, shown by the more efficient interaction of the RBD domain of NS1 with the active form of Akt compared to the effector domain of NS1 (EFF) in this assay. The inactive form of Akt failed to interact with either the EFF or the RBD of NS1 in this assay.

\subsection{NS1 enhanced the Akt phosphorylation in in vitro kinase assays.}

We next determine whether the interaction between NS1 and Akt can functionally affect the levels of Akt phosphorylation. Therefore, we compared the Akt phosphorylation and its substrate in $293 \mathrm{~T}$ cells transfected with either NS1 or a control vector (Sham). The levels of Akt phosphorylation at Thr308 increased in the presence of NS1. The increased Akt phosphorylation was correlated with phosphorylation of BAD and FOXO, well known substrates of Akt (Fig. 2A). 
We performed in vitro Akt kinase assays to determine whether the NS1-Akt interaction affects the augmentation of Akt phosphorylation and its substrate. In vitro Akt kinase assays demonstrated that increasing the amount of recombinant NS1 enhanced the levels of Akt phosphorylation at Ser473, Thr308, and phospho-GSK3 $\beta$, a substrate of Akt in a dose-dependent manner. The results support the notion that NS1 directly enhances the Akt activation (Fig. 2B).

Precedent evidence suggests that dimerization plays a critical role in enhancing the phosphorylation of intra-cellular molecules [6]. Recent reports have demonstrated that the amino acid position at Trp187(W187) of NS1 protein mediates its dimerization [9]. Therefore, we next verified the dimerization of NS1 protein on the induction of Akt phosphorylation. In in vitro kinase assays, wild-type NS1 enhanced the Akt phosphorylation more efficiently than the dimerization defective mutant NS1 (W187A, [9]) (Fig. 2C). The observation suggested that dimerization of NS1 plays a critical role in the induction of Akt phosphorylation.

\subsection{Co-localization of phospho-Akt with NS1 by confocal microscopy}

We showed that NS1 preferentially interacts with phosphorylated Akt (see Fig. 1D 
and E). Activated Akt is known to translocalize to the nucleus [8; 10]. Further, NS1, which harbors a pair of nuclear localizing signals, is known to be localized primarily within the nucleus[11;12]. Confocal microscopy was used to determine the co-localization of NS1 with phosphorylated Akt. Consistent with two nuclear localizing signals present within the NS1 protein, NS1 is predominantly localized within the nucleus during the mitotic phase. Furthermore, phosphorylated Akt interacted with NS1 mainly during the interphase of the cell cycle within the nucleus. In contrast, during the mitotic phase of the cell cycle, phosphoryated Akt failed to interact with the NS1 protein (Fig. 3).

Since the Akt-PH domain primarily mediated the interaction between Akt and NS1 (see Fig. 1C), we next examined whether ectopic introduction of the Akt-PH domain could alter the subcellular distribution/localization of the NS1 protein. Consistently, ectopic expression of the Akt-PH domain translocalized NS1 from the nucleus to the cytosole (data not shown).

\subsection{NS1 was phosphorylated at threonine residue at 215 in mass spectrometry.}

Since NS1 enhanced the Akt kinase activities, we next examined whether activated 
Akt may affect the phosphorylation levels of NS1. We performed in vitro Akt kinase assay using recombinant wild-type NS1 protein as a substrate and found that NS1 protein enhanced the levels of its phosphorylation in a time-dependent manner detected by Ser/Thr phospho-specific antibody (Fig. 4A). Akt activation consistently enhanced NS1 phosphorylation in 293 T cells (compare Fig. 4B). In order to define the precise Akt-induced phosphorylation site of NS1, mass spectrometric analysis was conducted. Transient transfection of Myr-Akt, a constitutively active form of Akt, with influenza NS1 induced the phosphorylation at Thr215 of NS1 protein, but failed to phosphorylate in the absence of Myr-Akt (Fig. 4C). However, it is important to note that since Thr215 of NS1 did not confirm the canonical Akt consensus phosphorylation site ( $\underline{\mathrm{R} X} \underline{\mathrm{R} X X} \underline{\mathrm{S}} / \mathrm{T})$, it is likely that secondary kinases accounted for the phosphorylation of Thr215 of NS1. 


\section{Discussion}

In recent years, influenza of H1N1 avian influenza viruses has emerged and now appears to be present in a sufficient number of countries to be called a pandemic and a major global health concern.

The influenza virus consists of a minus strand RNA virus and harbors 8 segmented RNA that encode 11 RNAs. Among the 11 proteins encoded by the minus strand RNA of avian influenza, segment 8 encodes NS1, a $26 \mathrm{kDa}$ protein that antagonizes the cellular immune response and is implicated in virulence [13]. The NS1 protein is the main IFN antagonistic factor for suppressing the initial expression of IFN- $\beta[1 ; 14]$ Hence, the influenza virus is considered to evade both innate and adaptive immunity via the NS1 protein. Viral NS1 is shown to activates PI3K signaling pathway as one of the major downstream effectors to enhance efficient replication[5; 15].

Consistent with the presence of a pair of nuclear localizing signals in the NS1, we used confocal microscopy to demonstrate that the NS1 is essentially localized within the nucleus. The observation could support the previous report that the function of the nuclear NS1 preferentially blocks the nucleoplasmic transport of the colliner RNP 8 transcript in an RNA-binding-dependent manner[16]. In co-immunoprecipitation assays and GST-pull down assays, we showed that NS1 protein preferentially interacted with 
activated Akt. The observation is consistent with previous report that activated Akt is translocalized to the nucleus $[8 ; 10]$. Furthermore, we showed that NS1 protein is co-localized with phosphorylated Akt predominantly within the nucleus during the mitotic phase, but is diffusely distributed in the interphase of the cell cycle by confocal microscopy. The nuclear function of Akt activation includes the regulation of the cell cycle progression through stabilization of cyclin $\mathrm{D}$ and inhibition of the $\mathrm{p} 27^{\mathrm{Kip} 1}$ protein via phosphorylation of 4E-BP1 and its dissociation from the mRNA cap-binding protein, eIF4E[6]. The physiological nature of the co-localization of NS1 with Akt during the interphase of the cell cycle remains to be determined, however, ectopic introduction of NS1, which enhances the accumulation of the S phase of the cell cycles, may account for this observation.

Using mass spectrometry, in the presence of Myr-Akt, a constitutively active form of Akt, we determined that Thr215 of NS1 was the primary phosphorylation site. Since Thr215 of NS1 ( SSNENGRPPLTPK ) did not conserve canonical phosphorylation motifs for Akt ( $\underline{\mathrm{R} X} \underline{\mathrm{RXX}} \underline{\mathrm{S}} / \mathrm{T}$ ), the phosphorylation at Thr215 of NS1 is likely to be mediated through secondary kinases mediated through Akt, such as P-ERK or cdk, for the induction of phosphorylation[17]. Consistent with the previous report that Myr-Akt induces activation of ERK and/or cdk [18; 19], it is likely that Thr215 phosphorylation 
is induced by indirect effect of these kinases mediated through the activation of Akt.

Although the precise molecular mechanisms as to how NS1 induces PI3K activation remain to be determined, NS1 has been shown to form a complex with $p 85 \beta$ subunit of PI3K to activate downstream signals[5; 15]. SH3-binding motif 1 in the influenza A virus NS1 protein is shown to be essential for the activation of the PI3K/Akt signaling pathway. Functionally, NS1 enhances intra-cellular signals to mediate anti-apoptotic responses and activate the signaling pathway to ensure efficient virus replication[20]. It is plausible that activation of PI3K/Akt pathway is beneficial for virus replication because it inhibits virus-induced apoptosis through phosphorlylation of caspase-9[21]. We cannot completely rule out the possibility that Akt kinase activity is activated via PI3K, however, dose-dependent escalation of Akt kinase activities observed in in vitro Akt kinase assays together supported that NS1 could directly target and interact with Akt and hence, enhances Akt kinase activity.

The current work will help to provide further understanding of the role of NS1 in human influenza infection for the development of new antiviral drugs by modulating the NS1-Akt functional interaction against the influenza virus infection. 


\section{Conflict of interest}

The authors declare that we do not have any financial interest related to the work described in this manuscript.

\section{Acknowledgements}

We thank Drs, J. Testa and T. Franke for valuable reagents. M.N. and F.S. are supported by Grant-in-Aid from the Japanese Ministry Education, the Naito, and The Suhara Memorial Foundation. 


\section{References}

[1] B.G. Hale, R.E. Randall, J. Ortin, and D. Jackson, The multifunctional NS1 protein of influenza A viruses. J Gen Virol 89 (2008) 2359-76.

[2] Z. Chen, Y. Li, and R.M. Krug, Chimeras containing influenza NS1 and HIV-1 Rev protein sequences: mechanism of their inhibition of nuclear export of Rev protein-RNA complexes. Virology 241 (1998) 234-50.

[3] D. Jackson, M.J. Killip, C.S. Galloway, R.J. Russell, and R.E. Randall, Loss of function of the influenza A virus NS1 protein promotes apoptosis but this is not due to a failure to activate phosphatidylinositol 3-kinase (PI3K). Virology 396 (2010) 94-105.

[4] B.G. Hale, and R.E. Randall, PI3K signalling during influenza A virus infections. Biochem Soc Trans 35 (2007) 186-7.

[5] C. Ehrhardt, and S. Ludwig, A new player in a deadly game: influenza viruses and the PI3K/Akt signalling pathway. Cell Microbiol 11 (2009) 863-71.

[6] M. Noguchi, V. Ropars, C. Roumestand, and F. Suizu, Proto-oncogene TCL1: more than just a coactivator for Akt. Faseb J 21 (2007) 2273-84.

[7] R. Murayama, Y. Harada, T. Shibata, K. Kuroda, S. Hayakawa, K. Shimizu, and T. Tanaka, Influenza A virus non-structural protein 1 (NS1) interacts with cellular multifunctional protein nucleolin during infection. Biochem Biophys Res Commun 362 (2007) 880-5.

[8] F. Suizu, Y. Hiramuki, F. Okumura, M. Matsuda, A.J. Okumura, N. Hirata, M. Narita, T. Kohno, J. Yokota, M. Bohgaki, C. Obuse, S. Hatakeyama, T. Obata, and M. Noguchi, The E3 ligase TTC3 facilitates ubiquitination and degradation of phosphorylated Akt. Dev Cell 17 (2009) 800-10. 
[9] S. Xia, and J.D. Robertus, X-ray structures of NS1 effector domain mutants. Arch Biochem Biophys 494 (2010) 198-204.

[10] M. Andjelkovic, D.R. Alessi, R. Meier, A. Fernandez, N.J. Lamb, M. Frech, P. Cron, P. Cohen, J.M. Lucocq, and B.A. Hemmings, Role of translocation in the activation and function of protein kinase B. J Biol Chem 272 (1997) 31515-24.

[11] K. Melen, L. Kinnunen, R. Fagerlund, N. Ikonen, K.Y. Twu, R.M. Krug, and I. Julkunen, Nuclear and nucleolar targeting of influenza A virus NS1 protein: striking differences between different virus subtypes. J Virol 81 (2007) 5995-6006.

[12] J. Schneider, and T. Wolff, Nuclear functions of the influenza A and B viruses NS1 proteins: do they play a role in viral mRNA export? Vaccine 27 (2009) 6312-6.

[13] D. Jackson, M.J. Hossain, D. Hickman, D.R. Perez, and R.A. Lamb, A new influenza virus virulence determinant: the NS1 protein four C-terminal residues modulate pathogenicity. Proc Natl Acad Sci U S A 105 (2008) 4381-6.

[14] K. Haye, S. Burmakina, T. Moran, A. Garcia-Sastre, and A. Fernandez-Sesma, The NS1 protein of a human influenza virus inhibits type I interferon production and the induction of antiviral responses in primary human dendritic and respiratory epithelial cells. J Virol 83 (2009) 6849-62.

[15] B.G. Hale, D. Jackson, Y.H. Chen, R.A. Lamb, and R.E. Randall, Influenza A virus NS1 protein binds p85beta and activates phosphatidylinositol-3-kinase signaling. Proc Natl Acad Sci U S A 103 (2006) 14194-9.

[16] U. Garaigorta, and J. Ortin, Mutation analysis of a recombinant NS replicon shows that influenza virus NS1 protein blocks the splicing and nucleo-cytoplasmic transport of its own viral mRNA. Nucleic Acids Res 35 (2007) 4573-82.

[17] B.G. Hale, A. Knebel, C.H. Botting, C.S. Galloway, B.L. Precious, D. Jackson, R.M. 
Elliott, and R.E. Randall, CDK/ERK-mediated phosphorylation of the human influenza A virus NS1 protein at threonine-215. Virology 383 (2009) 6-11.

[18] E.R. Lee, J.Y. Kim, Y.J. Kang, J.Y. Ahn, J.H. Kim, B.W. Kim, H.Y. Choi, M.Y. Jeong, and S.G. Cho, Interplay between PI3K/Akt and MAPK signaling pathways in DNA-damaging drug-induced apoptosis. Biochim Biophys Acta 1763 (2006) 958-68.

[19] D. Ramljak, C.M. Coticchia, T.G. Nishanian, M. Saji, M.D. Ringel, S.D. Conzen, and R.B. Dickson, Epidermal growth factor inhibition of c-Myc-mediated apoptosis through Akt and Erk involves Bcl-xL upregulation in mammary epithelial cells. Exp Cell Res 287 (2003) 397-410.

[20] C. Ehrhardt, T. Wolff, S. Pleschka, O. Planz, W. Beermann, J.G. Bode, M. Schmolke, and S. Ludwig, Influenza A virus NS1 protein activates the PI3K/Akt pathway to mediate antiapoptotic signaling responses. J Virol 81 (2007) 3058-67.

[21] Y.K. Shin, Y. Li, Q. Liu, D.H. Anderson, L.A. Babiuk, and Y. Zhou, SH3 binding motif 1 in influenza A virus NS1 protein is essential for PI3K/Akt signaling pathway activation. J Virol 81 (2007) 12730-9. 
Fig. 1 Influenza virus NS1 interacts with the Akt PH domain.

A. Co-immunoprecipitation assays using 293T cells transfected with HA-tagged human Akt2 with Flag-tagged NS1. Akt interacted with NS1 in this experiment. The results were consistent in three independent experiments.

B. Endogenous Akt antibody co-immunoprecipitated with NS1 protein (lane4, second panel from the top). Note that no NS1 protein could be immunoprecipitated by the control mouse IgG (lane1, top panels). Expression of the NS1 protein and endogenous Akt of the PR8-infected cells were shown in the lower panels as internal controls.

C. To determine the interaction domains between Akt with NS1 protein, co-immunoprecipitation assays were performed using 293T cells transfected with HA-tagged human full length Akt2 (WT), PH-domain of Akt (PH), or the C-terminal domain (C-term) of Akt with Flag-tagged NS1 protein. The PH-domain of Akt interacted with NS1, as shown by co-immunoprecipitation assays. 
D. To compare and further determine the interacting domain of NS1 with Akt, we performed GST pull-down assays. The RBD domain of NS1 (AA1 73) mediated the interaction between the activated and inactivated forms of Akt (active/inactive Akt, \#14-276 active Akt1/PKB $\alpha, \# 14-279$ inactive Akt1/PKB $\alpha$, Upstate Biotech, USA) with NS1. NS1 interacted with the active form of Akt but not with the inactive form of Akt in this assay.

E. To compare the effect of the Akt phosporylation for the NS1 interaction, we performed co-immunoprecipitation assays using wild type-Akt and DM-Akt, in which both Thr308 and Ser473 were substituted into Alanine using transient transfected $293 \mathrm{~T}$ cells. NS1 interacted with wild type Akt more efficiently than the DM-Akt, suggesting that phosphorylation of Akt is required for the NS1-Akt interaction. 
Fig. 2 NS1 enhances Akt phosphorylation in both cellular and in vitro kinase assays.

A. In transient transfection of NS1 into 293T cells, levels of Akt phosphorylation (at Thr308) increased, which is correlated with the phosphorylation levels of BAD and FOXO, well known substrates for Akt.

B. In order to determine the augmentation of Akt, phosphorylation was mediated through Akt, and in vitro kinase assay was performed using recombinant NS1 protein. Levels of both Akt phosphorylation at Ser473, Thr308, and phospho-GSK3 3 , a substrate of Akt were increased in a dose-dependent manner, in in vitro kinase assay. The results supported that NS1 directly enhances the Akt activation in in vitro kinase assays. We adjusted the total amount of the protein constant in in vitro kinase assays by adding a decreasing amount of GST control protein (see the top panel).

C. NS1 protein is known to form a dimer via amino acid at W187 of NS1 [9]. In order to examine the effect of dimerization of NS1 on the augmentation of Akt 
phosphorylation, we compared the levels of phosphorylation of Akt and its substrate in in vitro kinase assays. Wild type Akt enhanced the Akt phosphorylation more efficiently than W187A-NS1 in in vitro kinase assay, suggesting that dimerization of NS1 plays an important role in Akt phosphorylation. 
Fig. 3. Co-localization of phospho-Akt with NS1 in the interphase of the cell cycle, but not in the mitotic phase

We showed that NS1 preferentially interacted with the phosphorylated form of Akt (see Fig. $1 \mathbf{D}$ and E). A pair of nuclear localizing signals present localized NS1 protein within the nucleus. In order to define the interaction of phosphorylated Akt with NS1 in the nucleus, a confocal microscope was utilized with DsRed-tagged NS1. As shown in the upper panels, phosphorylated Akt (green) co-localized with the NS1 protein in HeLa cells during the interphase of the cell cycle, predominantly within the nucleus, with a speckled pattern distribution (shown by the arrows). In contrast, during the mitotic phase of the cell cycle, NS1 protein is more diffusely distributed throughout the nucleus and no longer co-localized with phosphorylated Akt (lower panels). 
Fig. 4. Myr-Akt enhances the phosphorylation of T215 in mass spectrometry analysis.

A. In vitro Akt kinase assays were performed using recombinant wild-type NS1 protein as a substrate. In the presence of Akt, recombinant NS1 protein clearly enhanced its levels of phosphorylation in a time-dependent manner, as detected by phospho-specific antibody (lanes 2 and 3).

B. 293T cells were transfected with wild type NS1 and activation of Akt was induced. Akt activation enhanced NS1 phosphorylation, as detected by phospho-specific antibody (compare bottom panel, lanes 1 and 2, unstimulated and stimulated cells).

C. In order to define the Akt-induced phosphorylation of NS1 protein, mass spectrometry analysis was conducted. Flag-tagged NS1 was transfected into 293T cells, along with Myr-Akt, a constitutive active form of Akt, by the calcium phosphate method. Immunoprecipitated Flag-NS1 was trypsinized in-gel to identify the phosphorylation sites using MS/MS analysis by LXQ (Thermo Fisher) equipped with nano-flow LC Magic (Michrom Bioresources, Inc.). In the presence of 
Myr-Akt, but not in the absence of Myr-Akt transfected cells (sham), the influenza

NS1 protein enhanced the phosphorylation at position Threonine 215. 
Figure 1
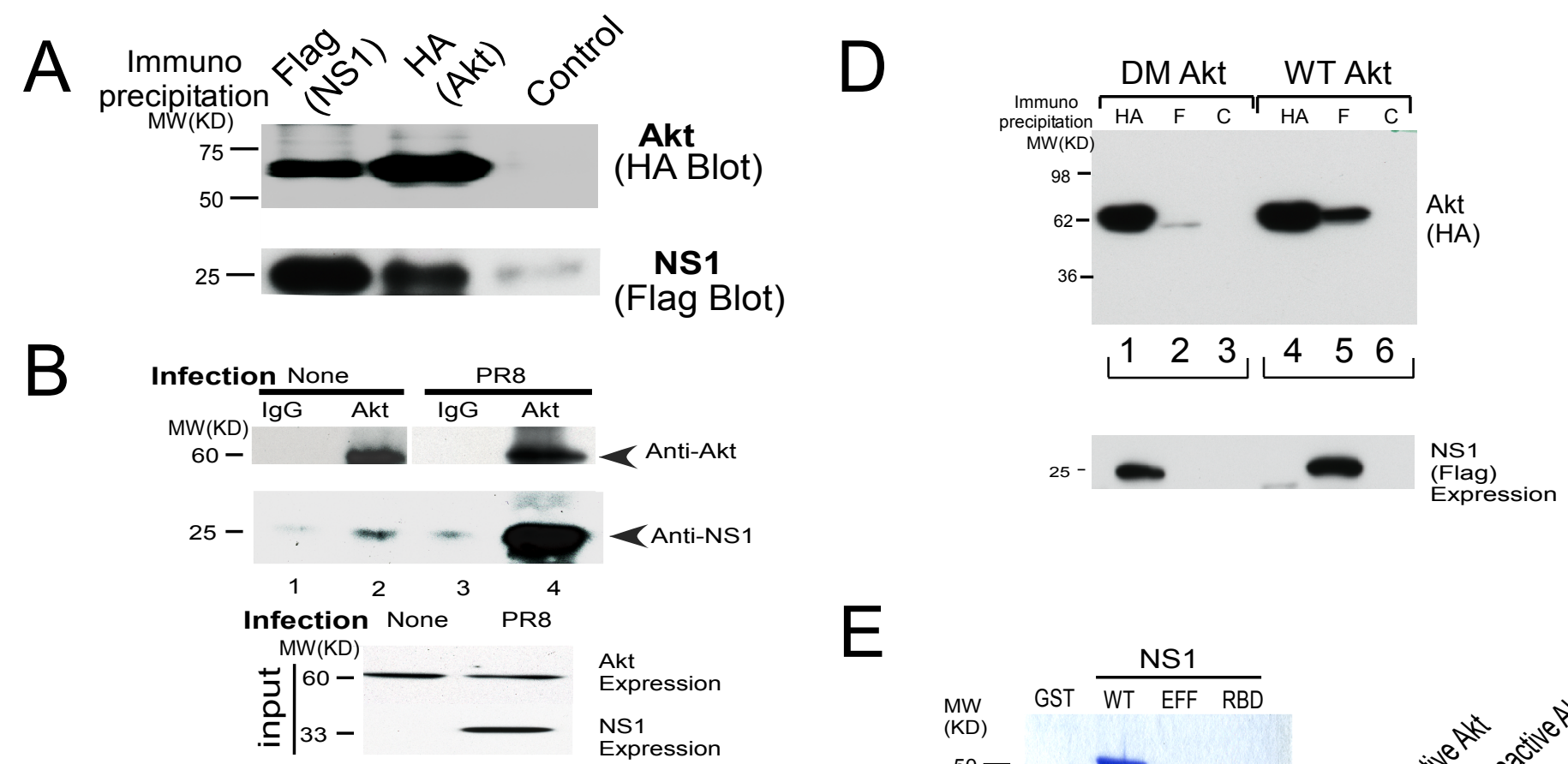

C

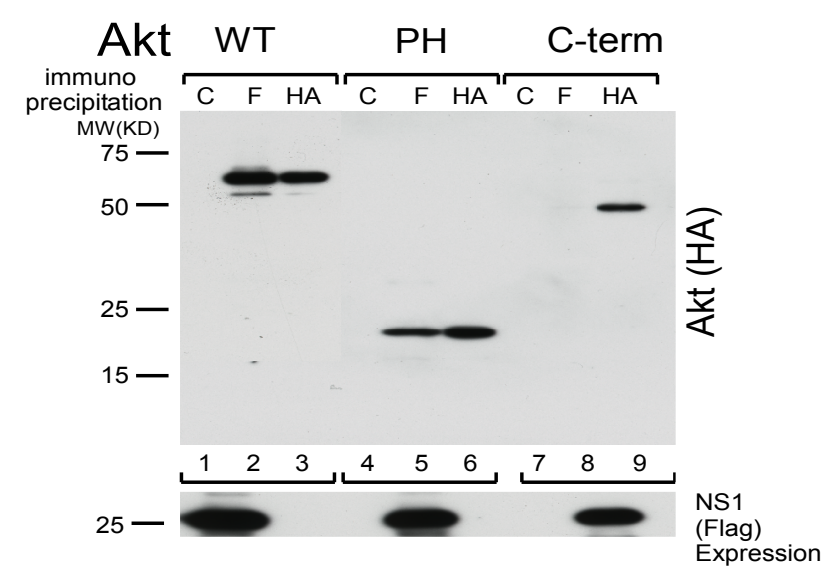

E
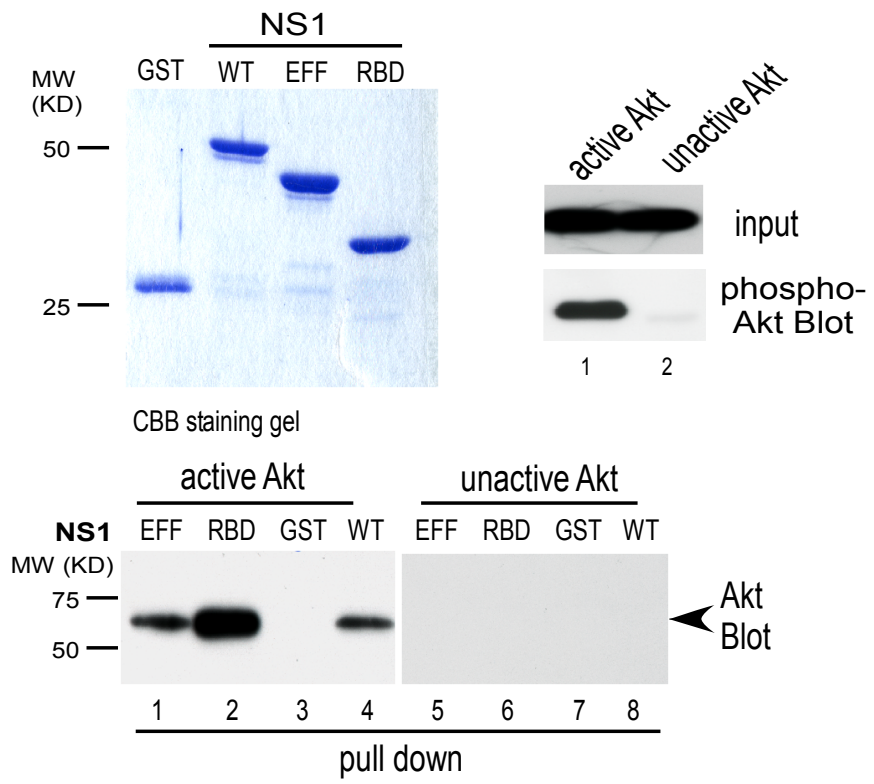
Figure 2

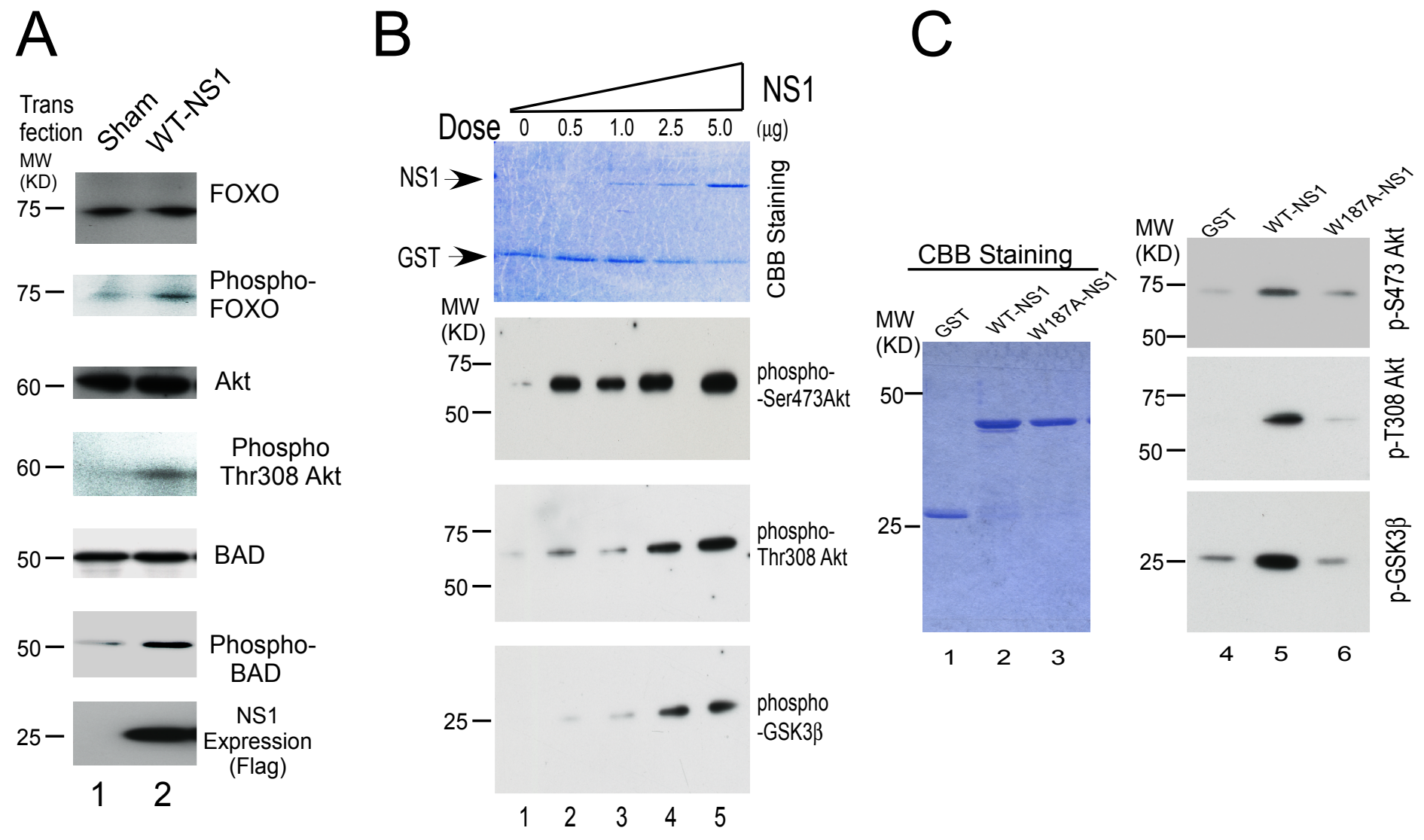


Figure 3

DsRed NS1

Interphase

Mitotic phase
phospho-Akt

(Ser473)
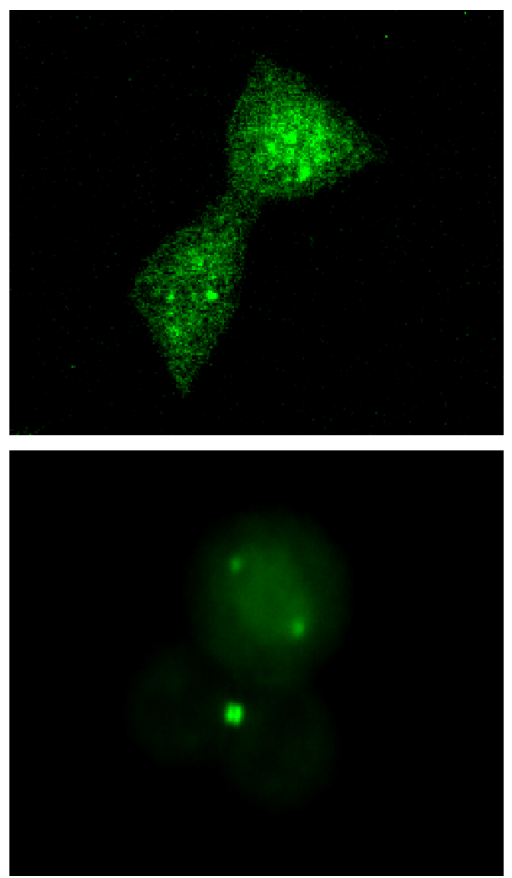

Merged

(+ DAPI)
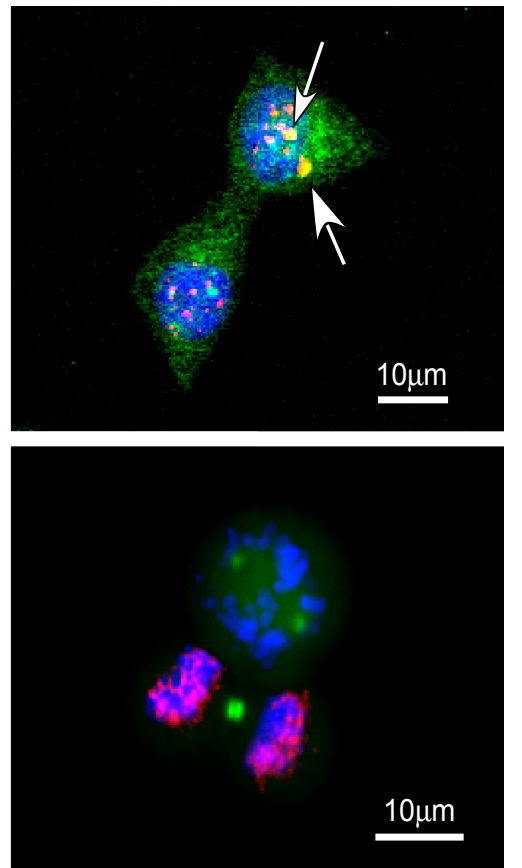
Figure 4

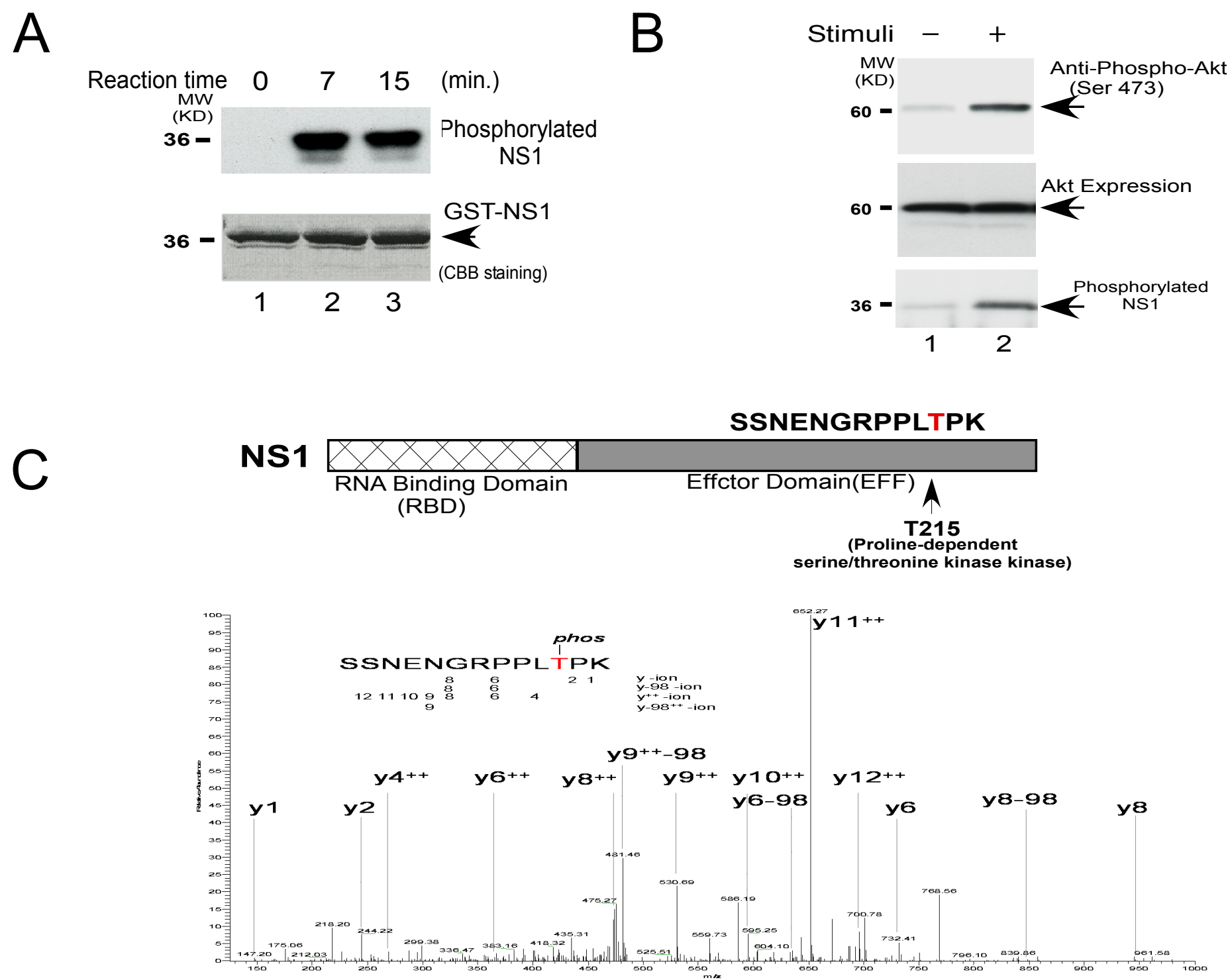




\section{Supplementary Material}

\section{Antibodies used in this study:}

anti-HA (12CA5, \#1666606, Roche Diagnostics, Inc.), anti-Flag (M2)-peroxidase

(\#A8592, Sigma), anti-Flag Affinity gel, (\#2220, Sigma), anti-Akt (\# 9272,or \#2966, Cell Signaling) , anti-phospho Ser473/Thr308 Akt (\#4051 for Ser 473 and \#9275 for Thr308, Cell Signaling), anti-phospho-Ser/Thr antibody (\#9614, Cell Signaling), anti-NS1 antibody vC20 (sc-17596, SantaCruz), anti-FKHR (\#9461, Cell Signaling), anti-phospho FKHR (\#9462, Cell Signaling), DAPI (4',6-diamidino-2-phenylindole \#D9564, Sigma), and mouse IgG 1 (\#x0931, Dako). 